\title{
Nonuniform Multiconductor Transmission Line Analysis by a Two-Step Perturbation Technique
}

\author{
Mykola Chernobryvko, Daniël De Zutter, Fellow, IEEE and Dries Vande Ginste, Senior Member, IEEE.
}

\begin{abstract}
A two-step perturbation technique to model nonuniform multiconductor transmission lines in the frequency domain is presented. In this method nonuniformities are treated as perturbations with respect to the nominal uniform multiconductor line. Starting from the Telegrapher's equations and applying two consecutive perturbations steps, at each step, we obtain secondorder ordinary differential equations with distributed source terms. Solving these equations together with the appropriate boundary conditions provides the sought-for voltages and currents along the interconnect structure. The method is validated by means of a frequency domain analysis of a ten conductor microstrip line with random uniformities, confirming its accuracy and efficiency. Additionally, the time domain accuracy and efficiency is demonstrated by means of a high-speed packaging nonuniform interconnect with six signal conductors.
\end{abstract}

Index Terms-Interconnect modeling, nonuniform multiconductor transmission line (NMTL), perturbation, Telegrapher's equations, transient analysis.

\section{INTRODUCTION}

$\mathbf{N}$ ONUNIFORM lines (NMTLs) multiconductor transmission interconnections in various microwave a to the increasing density, operation speed and complexity of modern integrated circuits, physical effects such as delay, ringing, distortion, and crosstalk cannot be neglected and must be captured properly as frequency increases. Moreover, skin, proximity, edge, and roughness effects can lead to signal integrity problems at high frequencies [1]. However, the analytical solution of differential equations describing the behavior of NMTLs with varying per-unit-length (p.u.1.) parameters along the line are not available for the general case.

Recently, several methods for analyzing NMTLs have been proposed in both time and frequency domains. The straightforward way to perform the analysis is to approximate an NMTL as a cascade of discrete uniform transmission lines [2], [3]. However, many segments have to be used in order to get an accurate solution. One of the most commonly used tools to obtain a transient response is the inverse fast Fourier transform [4]. Nevertheless, it requires very many data points to avoid aliasing errors when very fast signals are studied. The method of characteristics [5]-[8], which discretizes both time and distance, can also be applied to obtain transients. Unfortunately, the technique becomes inefficient to account for frequency-dependent p.u.l. parameters. Another technique

M. Chernobryvko, D. Vande Ginste and D. De Zutter are with the Electromagnetics Group, Department of Information Technology, Ghent University/iMinds, St. Pietersnieuwstraat 41, 9000 Ghent, Belgium (e-mail: mykola.chernobryvko@intec.ugent.be). transforms the Telegrapher's equations into algebraic equations in frequency or in time domain using wavelet expansions [9][11]. The accuracy of the method depends on the number of components of the wavelet basis. Also, its complexity grows significantly with the number of signal conductors. Full-wave simulations of NMTLs based on the method of moments (MoM) [12], finite-difference time-domain (FDTD) technique [13]-[15], finite elements method (FEM) [16] or differential quadrature method (DQM) [17], [18] provide results with high accuracy, but the computational expenses of the fullwave techniques considerably exceed those of the quasi-TM approach.

In this paper, we propose a two-step perturbation technique to analyze NMTLs in the frequency domain. Whereas only nonuniform single and differential lines could be treated in [19], this paper presents the general theory for the case with $N$ signal conductors. The perturbation approach can be applied to NMTLs for which the cross-sectional properties vary in an arbitrary way. We perform the analysis using the well-known RLGC-matrix description for transmission lines in the quasi-TM regime [20]. The NMTL is represented as a uniform multiconductor transmission line with perturbations describing the nonuniformities. This uniform interconnect is considered to be the nominal structure in our approach. First, the nominal voltages and currents are found as a solution of the classical Telegrapher's equations. Next, in the first perturbation step, we obtain the first-order perturbation values of voltages and currents solving a similar set of Telegrapher's equations with additional distributed voltage and current sources. These source terms depend on the nominal voltages and currents and on the deviation of p.u.l. parameters from their nominal values in each point along the line. As was already shown in [19], a second perturbation step is needed to significantly improve the accuracy of our technique. To demonstrate the accuracy and efficiency of the technique, two examples are worked out in detail. First, a nonuniform transmission line with ten signal conductors, for which the cross-sectional properties change randomly, is investigated in the frequency domain. The second example is a high-speed packaging interconnect example composed of six nonuniform lines. To perform the analysis of the second structure in the time domain, the results of the perturbation approach are imported into Agilent's ADS framework and compared to the full-wave solution of ADS.

The outline of this paper is as follows. Section II details the two-step perturbation technique for nonuniform transmission lines with $N$ signal conductors. The details of the firstorder perturbation solution are considered in Section II-B. The theory is validated and illustrated in Section III. Section IV 
summarizes our work and conclusions.

\section{FORMALISM FOR GENERAL NONUNIFORM MULTICONDUCTOR LINES}

We analyse NMTLs within the framework of the quasiTM approach and in the frequency domain (with the $e^{j \omega t}$ dependency suppressed). Consider voltage and current $N \times 1$ column vectors $\mathcal{V}$ and $\mathcal{I}$, holding the $N$ voltages and $N$ currents along the lines where $N$ is the number of signal conductors and with the voltages defined with respect to a common reference conductor (conductor $N+1$ ). To simplify the notation, we work with $N \times N$ complex p.u.l. inductance $\mathcal{L}$ and capacitance $\mathcal{C}$ matrices, i.e. the p.u.l. resistance $\mathcal{R}$ and conductance $\mathcal{G}$ are understood to be part of $\mathcal{L}$ and $\mathcal{C}$ $\left(\mathcal{L}=\mathcal{L}+\frac{\mathcal{R}}{j \omega}\right.$ and $\left.\mathcal{C}=\mathcal{C}+\frac{\mathcal{G}}{j \omega}\right)$. Our starting point is the well-known Telegrapher's equations:

$$
\begin{aligned}
& \frac{d \mathcal{V}(z)}{d z}=-j \omega \mathcal{L}(z) \mathcal{I}(z), \\
& \frac{d \mathcal{I}(z)}{d z}=-j \omega \mathcal{C}(z) \mathcal{V}(z),
\end{aligned}
$$

with $z$ being the signal propagation direction. To formulate a perturbation technique, the following expansions are introduced:

$$
\begin{aligned}
& \mathcal{V}(z)=\tilde{\mathcal{V}}(z)+\Delta \mathcal{V}_{1}(z)+\Delta \mathcal{V}_{2}(z)+\ldots, \\
& \mathcal{I}(z)=\tilde{\mathcal{I}}(z)+\Delta \mathcal{I}_{1}(z)+\Delta \mathcal{I}_{2}(z)+\ldots, \\
& \mathcal{C}(z)=\tilde{\mathcal{C}}+\Delta \mathcal{C}(z) \\
& \mathcal{L}(z)=\tilde{\mathcal{L}}+\Delta \mathcal{L}(z) .
\end{aligned}
$$

The leading terms of the series expansions (3), i.e. the voltage $\tilde{\mathcal{V}}(z)$ and current $\tilde{\mathcal{I}}(z)$, are labeled as the unperturbed values. The remaining terms are perturbations of order one, two, etc. $\mathcal{C}(z)$ and $\mathcal{L}(z)$ in (3) are simply written as the sum of a constant part and a place-dependent part. Here, $\tilde{\mathcal{C}}$ and $\tilde{\mathcal{L}}$ are the unperturbed values. $\Delta \mathcal{C}(z)$ and $\Delta \mathcal{L}(z)$ are the variations of the capacitance and inductance along the line, which remain after subtracting the constant martrices $\tilde{\mathcal{C}}$ and $\tilde{\mathcal{L}}$ from $\mathcal{C}(z)$ and $\mathcal{L}(z)$ respectively. Remark that $\tilde{\mathcal{C}}$ and $\tilde{\mathcal{L}}$ are not necessarily the mean values of $\mathcal{C}$ and $\mathcal{L}$ over the line. We only suppose that $\Delta \mathcal{C}(z)$ and $\Delta \mathcal{L}(z)$ are small enough with respect to $\tilde{\mathcal{C}}$ and $\tilde{\mathcal{L}}$. To simplify notations, the $z$-dependence between the brackets will be dropped in the sequel. For the unperturbed quantities we have:

$$
\begin{aligned}
& \frac{d \tilde{\mathcal{V}}}{d z}=-j \omega \tilde{\mathcal{L}} \tilde{\mathcal{I}}, \\
& \frac{d \tilde{\mathcal{I}}}{d z}=-j \omega \tilde{\mathcal{C}} \tilde{\mathcal{V}},
\end{aligned}
$$

while the perturbations of order one and two satisfy

$$
\begin{aligned}
& \frac{d \Delta \mathcal{V}_{1}}{d z}=-j \omega \tilde{\mathcal{L}} \Delta \mathcal{I}_{1}-j \omega \Delta \mathcal{L} \tilde{\mathcal{I}}, \\
& \frac{d \Delta \mathcal{I}_{1}}{d z}=-j \omega \tilde{\mathcal{C}} \Delta \mathcal{V}_{1}-j \omega \Delta \mathcal{C} \tilde{\mathcal{V}}, \\
& \frac{d \Delta \mathcal{V}_{2}}{d z}=-j \omega \tilde{\mathcal{L}} \Delta \mathcal{I}_{2}-j \omega \Delta \mathcal{L} \Delta \mathcal{I}_{1}, \\
& \frac{d \Delta \mathcal{I}_{2}}{d z}=-j \omega \tilde{\mathcal{C}} \Delta \mathcal{V}_{2}-j \omega \Delta \mathcal{C} \Delta \mathcal{V}_{1} .
\end{aligned}
$$

\section{A. The unperturbed problem}

Let us now summarize what is relevant to the solution of the unperturbed problem. Both $\tilde{\mathcal{V}}$ and $\tilde{\mathcal{I}}$ satisfy a wave equation:

$$
\begin{aligned}
& \frac{d^{2} \tilde{\mathcal{V}}}{d z^{2}}+\omega^{2}(\tilde{\mathcal{L}} \tilde{\mathcal{C}}) \tilde{\mathcal{V}}=0, \\
& \frac{d^{2} \tilde{\mathcal{I}}}{d z^{2}}+\omega^{2}(\tilde{\mathcal{C}} \tilde{\mathcal{L}}) \tilde{\mathcal{I}}=0 .
\end{aligned}
$$

To solve (10) and (11), the voltages are expanded in terms of the eigenvectors $V_{i}$ of $\tilde{\mathcal{L}} \tilde{\mathcal{C}}$ and the currents in terms of the eigenvectors $I_{i}$ of $\tilde{\mathcal{C}} \tilde{\mathcal{L}}$ :

$$
\begin{aligned}
& \tilde{\mathcal{V}}=\sum_{i=1}^{N} \alpha_{i} V_{i}, \\
& \tilde{\mathcal{I}}=\sum_{i=1}^{N} \tilde{\alpha}_{i} I_{i} .
\end{aligned}
$$

From now on we will systematically introduce vector and matrix notations to avoid working with individual eigenvectors. Let us store the coefficients $\alpha_{i}$ in the $N \times 1$ column vector $\tilde{\mathbf{v}}$ and likewise, the coefficients $\tilde{\alpha}_{i}$ in the column vector $\tilde{\mathbf{i}}$. The eigenvectors $V_{i}$ are collected in a $N \times N$ matrix $\mathbf{T}$, column $i$ of which is $V_{i}$ and the $I_{i}$ 's are similarly collected in $\mathbf{S}$. Hence, (12) and (13) can be concisely written as

$$
\begin{aligned}
& \tilde{\mathcal{V}}=\mathbf{T} \tilde{\mathbf{v}} \\
& \tilde{\mathcal{I}}=\mathbf{S} \tilde{\mathbf{i}} .
\end{aligned}
$$

As proven in Appendix A (and is well-known), the eigenvectors of voltages and currents are biorthogonal and hence, with proper normalization, we can assert that $\mathbf{T}^{T} \mathbf{S}=\mathbf{S}^{T} \mathbf{T}=\mathcal{I}_{N}$, where $\mathcal{I}_{N}$ is the $N \times N$ unit matrix. The critical reader will remark that this orthonormalization does not uniquely determine the eigenvectors as a particular voltage eigenvector can be multiplied by a constant, provided the corresponding current eigenvector is divided by that same factor. We will not pursue this issue here but remark that in the end, the actual voltages and currents as given by (12) and (13) remain unchanged.

At this point, it is very important to remark that the above reasoning (and further properties used in this paper and proven in Appendix A) are only valid provided all eigenvalues are distinct. If this is not the case, due care has to be taken to still obtain a diagonal modal impedance matrix. This is possible when using so-called "generalized associated eigenvectors". For more details we refer the reader to [21] and [22]. The examples treated in the present paper are of such a nature that the eigenvalues are distinct, which is also what is assumed in the further derivation of the theory below. In [21] and [22] the reader will find the necessary material to extend the theory to the more general case.

Furthermore (also proven in Appendix A), the eigenvalues $\lambda_{i}$ of voltage and current eigenvectors are identical. For further use, we will need a diagonal $N \times N$ matrix $\Lambda$, with diagonal elements $\lambda_{i}$. With this eigenvalue matrix, the eigenvector matrices $\tilde{\mathbf{v}}$ and $\tilde{\mathbf{i}}$ satisfy

$$
\begin{aligned}
& (\tilde{\mathcal{L}} \tilde{\mathcal{C}}) \mathbf{T}=\mathbf{T} \Lambda \\
& (\tilde{\mathcal{C}} \tilde{\mathcal{L}}) \mathbf{S}=\mathbf{S} \Lambda .
\end{aligned}
$$


Using the above and substituting (14) into (10) and (15) into (11) shows that:

$$
\begin{aligned}
& \frac{d^{2} \tilde{\mathbf{v}}}{d z^{2}}+\omega^{2} \Lambda \tilde{\mathbf{v}}=0, \\
& \frac{d^{2} \tilde{\mathbf{i}}}{d z^{2}}+\omega^{2} \Lambda \tilde{\mathbf{i}}=0 .
\end{aligned}
$$

Let us go back to (4), insert the eigenvector expansions (14) and (15) and project both sides of the equation on the current eigenvectors. This yields

$$
\frac{d \tilde{\mathbf{v}}}{d z}=-j \omega \mathbf{L} \tilde{\mathbf{i}}
$$

with the matrix $\mathbf{L}$ given by

$$
\mathbf{L}=\mathbf{S}^{T} \tilde{\mathcal{L}} \mathbf{S} .
$$

As proven in Appendix A, the current eigenvectors satisfy the orthogonality property $\left(I_{i}\right)^{T} \tilde{\mathcal{L}} I_{j}=0$ for $i \neq j$, hence, $\mathbf{L}$ is a diagonal matrix. Similarly, starting from (5) we arrive at

$$
\begin{aligned}
\frac{\tilde{d} \mathbf{i}}{d z} & =-j \omega \mathbf{C} \tilde{\mathbf{v}}, \\
\mathbf{C} & =\mathbf{T}^{T} \tilde{\mathcal{C}} \mathbf{T},
\end{aligned}
$$

with $\mathbf{C}$ a diagonal matrix as a consequence of the orthogonality property $\left(V_{i}\right)^{T} \tilde{\mathcal{C}} V_{j}=0$ for $i \neq j$ (see Appendix A). Futhermore, we have

$$
\mathbf{L C}=\mathbf{C L}=\Lambda .
$$

Let us now proceed by first solving (17) yielding

$$
\tilde{\mathbf{v}}=e^{-j \mathbf{K} z} \mathbf{A}+e^{+j \mathbf{K} z} \mathbf{B}
$$

with $\mathbf{K}=\omega \sqrt{\Lambda}$ and with $\mathbf{A}$ and $\mathbf{B}$ complex amplitude $N \times 1$ vectors. The matrix exponential and square root are welldefined as $\Lambda$ is a diagonal matrix. From (24), (19) and (23) it is found that

$$
\tilde{\mathbf{i}}=\mathbf{Z}_{m}^{-1}\left(e^{-j \mathbf{K} z} \mathbf{A}-e^{+j \mathbf{K} z} \mathbf{B}\right),
$$

with the (diagonal) modal impedance matrix $\mathbf{Z}_{m}$ given by

$$
\mathbf{Z}_{m}=\sqrt{\mathbf{L} \mathbf{C}^{-1}}=\sqrt{\mathbf{C}^{-1} \mathbf{L}} .
$$

In order to determine the actual values of $\mathbf{A}$ and $\mathbf{B}$ we have to impose the boundary conditions. For an NTML of length $l$ at $z=0$ and $z=l$, we impose that

$$
\begin{gathered}
\tilde{\mathcal{V}}(z=0)+\mathcal{Z}_{s} \tilde{\mathcal{I}}(z=0)=\mathcal{V}_{s}, \\
\tilde{\mathcal{V}}(z=l)-\mathcal{Z}_{L} \tilde{\mathcal{I}}(z=l)=0,
\end{gathered}
$$

where the currents are directed in the positive $z$-direction at both the source and load side and with $\mathcal{Z}_{s}$ and $\mathcal{Z}_{L}$ the $N \times N$ source side and load side impedance matrices resp. and with $\mathcal{V}_{s}$ the $N \times 1$ source column vector. At the source, (14), (15), (24), (25) and (27) show that

$$
\mathbf{T}(\mathbf{A}+\mathbf{B})+\mathcal{Z}_{s} \mathbf{S Z}_{m}^{-1}(\mathbf{A}-\mathbf{B})=\mathcal{V}_{s} .
$$

Left multiplication with $\mathbf{S}^{T}$ yields

$$
(\mathbf{A}+\mathbf{B})+\mathbf{Z}_{s} \mathbf{Z}_{m}^{-1}(\mathbf{A}-\mathbf{B})=\mathbf{V}_{s}
$$

where we have introduced the following quantities

$$
\begin{aligned}
\mathbf{Z}_{s} & =\mathbf{S}^{T} \mathcal{Z}_{s} \mathbf{S}, \\
\mathbf{V}_{s} & =\mathbf{S}^{T} \mathcal{V}_{s} .
\end{aligned}
$$

Note the similarity between (31) and (20). The $N \times 1$ voltage vector $\mathbf{V}_{s}$ is the original voltage vector $\mathcal{V}_{s}$ projected on the current eigenvectors. At the load, (14), (15), (24), (25) and (27) now show that

$$
\begin{array}{r}
\mathbf{T}\left(e^{-j \mathbf{K} l} \mathbf{A}+e^{+j \mathbf{K} l} \mathbf{B}\right) \\
-\mathcal{Z}_{L} \mathbf{S Z}_{m}^{-1}\left(e^{-j \mathbf{K} l} \mathbf{A}-e^{+j \mathbf{K} l} \mathbf{B}\right)=0 .
\end{array}
$$

Left multiplication with $\mathbf{S}^{T}$ gives

$$
\begin{array}{r}
\left(e^{-j \mathbf{K} l} \mathbf{A}+e^{+j \mathbf{K} l} \mathbf{B}\right) \\
-\mathbf{Z}_{L} \mathbf{Z}_{m}^{-1}\left(e^{-j \mathbf{K} l} \mathbf{A}-e^{+j \mathbf{K} l} \mathbf{B}\right)=0
\end{array}
$$

with

$$
\mathbf{Z}_{L}=\mathbf{S}^{T} \mathcal{Z}_{L} \mathbf{S}
$$

Finally, (30) and (34) yield the following set of equations for the unknown complex wave amplitudes:

$$
\begin{array}{r}
\left(\begin{array}{cc}
\mathcal{I}_{N}+\mathbf{Z}_{s} \mathbf{Z}_{m}^{-1} & \mathcal{I}_{N}-\mathbf{Z}_{s} \mathbf{Z}_{m}^{-1} \\
\left(e^{-j \mathbf{K} l}-\mathbf{Z}_{L} \mathbf{Z}_{m}^{-1} e^{-j \mathbf{K} l}\right) & \left(e^{+j \mathbf{K} l}+\mathbf{Z}_{L} \mathbf{Z}_{m}^{-1} e^{+j \mathbf{K} l}\right)
\end{array}\right)\left(\begin{array}{l}
\mathbf{A} \\
\mathbf{B}
\end{array}\right) \\
=\left(\begin{array}{c}
\mathbf{V}_{s} \\
0
\end{array}\right) .
\end{array}
$$

To emphasize the analogy with the single line problem, the above result is rewritten as

$$
\begin{array}{r}
\left(\begin{array}{cc}
\phi_{s} & \boldsymbol{\varphi}_{s} \\
\boldsymbol{\varphi}_{L} e^{-j \mathbf{K} l} & \phi_{L} e^{+j \mathbf{K} l}
\end{array}\right)\left(\begin{array}{l}
\mathbf{A} \\
\mathbf{B}
\end{array}\right) \\
=\left(\begin{array}{c}
\mathbf{V}_{s} \\
0
\end{array}\right),
\end{array}
$$

with

$$
\begin{aligned}
\boldsymbol{\phi} & =\mathcal{I}_{N}+\mathbf{Z} \mathbf{Z}_{m}^{-1}, \\
\varphi & =\mathcal{I}_{N}-\mathbf{Z} \mathbf{Z}_{m}^{-1},
\end{aligned}
$$

and where the subindex "s" or " $\mathrm{L}$ " is added to distinguish between the source and load impedance matrices resp. The product $\phi^{-1} \varphi$ represents a generalized reflection coefficient. To conclude this subsection, we would like to draw the attention to the fact that the modal impedance matrix $\mathbf{Z}_{m}$ is not uniquely defined. Indeed, the eigenvectors in the eigenvector matrix $\mathbf{v}$ are only defined up to a multiplicative constant, implying that $\mathbf{C}$ and $\mathbf{L}$ are also not uniquely defined. This does not influence the eigenvalues: they remain fixed. Going back to the original voltages $\tilde{\mathcal{V}}$ and currents $\tilde{\mathcal{I}}$, using (14), (15), (24) and (25), we readily deduce that $\tilde{\mathcal{V}}=\mathbf{Z}_{i n} \tilde{\mathcal{I}}$, with the input impedance matrix of the infinite multiconductor line given by

$$
\mathbf{Z}_{i n}=\mathbf{T} \mathbf{Z}_{m} \mathbf{T}^{T}
$$

Using (20), (22) and the fact that $\mathbf{S}^{T} \mathbf{T}=\mathbf{T}^{T} \mathbf{S}=\mathcal{I}_{N}$, one can prove that $\mathbf{Z}_{\text {in }}$ is indeed unique, as it should be. 


\section{B. The perturbed problem}

Let us now turn to the perturbations. Taking the $z$-derivative of (6) and using (7), we find that

$$
\frac{d^{2} \Delta \mathcal{V}_{1}}{d z^{2}}+\omega^{2}(\tilde{\mathcal{L}} \tilde{\mathcal{C}}) \Delta \mathcal{V}_{1}=-\omega^{2}(\tilde{\mathcal{L}} \Delta \mathcal{C}) \tilde{\mathcal{V}}-j \omega \frac{d}{d z}(\Delta \mathcal{L} \tilde{\mathcal{I}})
$$

Voltage and current perturbations of order one are also expanded in the corresponding eigenvectors as,

$$
\begin{aligned}
& \Delta \mathcal{V}_{1}=\sum_{i=1}^{N} \beta_{i} V_{i}=\mathbf{T} \mathbf{v}_{1}, \\
& \Delta \mathcal{I}_{1}=\sum_{i=1}^{N} \tilde{\beta}_{i} I_{i}=\mathbf{S i}_{1} .
\end{aligned}
$$

The $\beta_{i}$ and $\tilde{\beta}_{i}$ coefficients have been collected in the vectors $\mathbf{v}_{1}$ and $\mathbf{i}_{1}$ resp. Inserting these expansions into (40) and taking the proper orthogonality into account, shows that

$$
\frac{d^{2} \mathbf{v}_{1}}{d z^{2}}+\omega^{2} \Lambda \mathbf{v}_{1}=-\omega^{2}\left(\mathbf{S}^{T} \tilde{\mathcal{L}} \Delta \mathcal{C} \mathbf{T}\right) \tilde{\mathbf{v}}-j \omega \frac{d}{d z}\left[\left(\mathbf{S}^{T} \Delta \mathcal{L} \mathbf{T}\right) \tilde{\mathbf{i}}\right]
$$

Once differential equation (43) is solved for $\mathbf{v}_{1}$, (6) shows that $\mathbf{v}_{1}$ can be solved from

$$
\frac{d \mathbf{v}_{1}}{d z}=-j \omega\left[\mathbf{L i}_{1}+\left(\mathbf{S}^{T} \Delta \mathcal{L} \mathbf{S}\right) \tilde{\mathbf{i}}\right] .
$$

To simplify further calculations and analogous to (20) and (22), we introduce

$$
\begin{aligned}
\Delta \mathbf{L} & =\mathbf{S}^{T} \Delta \mathcal{L} \mathbf{S}, \\
\Delta \mathbf{C} & =\mathbf{T}^{T} \Delta \mathcal{C} \mathbf{T} .
\end{aligned}
$$

Contrary to $\mathbf{L}$ and $\mathbf{C}$, these matrices are not diagonal. With this notation, (43) and (44) become

$$
\begin{aligned}
\frac{d^{2} \mathbf{v}_{1}}{d z^{2}}+\omega^{2} \Lambda \mathbf{v}_{1} & =-\omega^{2} \mathbf{L} \Delta \mathbf{C} \tilde{\mathbf{v}}-j \omega \frac{d}{d z}(\Delta \mathbf{L} \tilde{\mathbf{i}}), \\
\frac{d \mathbf{v}_{1}}{d z} & =-j \omega\left(\mathbf{L i}_{1}+\Delta \mathbf{L} \tilde{\mathbf{i}}\right) .
\end{aligned}
$$

A particular solution to (46) can be found by applying the general theory for second-order differential equations with an arbitrary source term (see e.g. [23] or Appendix A of [24]),i.e.

$$
\begin{gathered}
-\frac{1}{2 j} \mathbf{K}^{-1} e^{-j \mathbf{K} z} \int_{0}^{z} e^{+j \mathbf{K} z^{\prime}}\left[-\omega^{2} \mathbf{L} \Delta \mathbf{C} \tilde{\mathbf{v}}-j \omega \frac{d}{d z^{\prime}}(\Delta \mathbf{L} \tilde{\mathbf{i}})\right] d z^{\prime} \\
+\frac{1}{2 j} \mathbf{K}^{-1} e^{+j \mathbf{K} z} \int_{0}^{z} e^{-j \mathbf{K} z^{\prime}}\left[-\omega^{2} \mathbf{L} \Delta \mathbf{C} \tilde{\mathbf{v}}-j \omega \frac{d}{d z^{\prime}}(\Delta \mathbf{L} \tilde{\mathbf{i}})\right] d z^{\prime} .
\end{gathered}
$$

The above expression can now be simplified by applying partial integration to the terms with the derivative $d / d z^{\prime}$. Careful calculations show that the resulting contributions of the upper limit of the integration interval (i.e. $z^{\prime}=z$ ) drop out, while the contributions of the lower limit of the integration interval (i.e. $z^{\prime}=0$ ) are of the form $\mathbf{C} e^{ \pm j \mathbf{K} z}$ with $\mathbf{C}$ a constant vector. Hence, it turns out that these contributions are solutions to the homogeneous equation, i.e. (46) without source. Consequently, we still have a valid particular solution if these contributions are dropped. The final result for $\mathbf{v}_{1}$, including an arbitrary solution to the homogeneous equation, then becomes

$$
\begin{array}{r}
\mathbf{v}_{1}=e^{-j \mathbf{K} z} \mathbf{P}+e^{+j \mathbf{K} z} \mathbf{Q} \\
-\frac{j \omega}{2} e^{-j \mathbf{K} z} \int_{0}^{z} e^{+j \mathbf{K} z^{\prime}}\left(\mathbf{Z}_{m} \Delta \mathbf{C} \tilde{\mathbf{v}}+\Delta \mathbf{L} \tilde{\mathbf{i}}\right) d z^{\prime} \\
+\frac{j \omega}{2} e^{+j \mathbf{K} z} \int_{0}^{z} e^{-j \mathbf{K} z^{\prime}}\left(\mathbf{Z}_{m} \Delta \mathbf{C} \tilde{\mathbf{v}}-\Delta \mathbf{L} \tilde{\mathbf{i}}\right) d z^{\prime}
\end{array}
$$

with $\mathbf{P}$ and $\mathbf{Q}$ as yet undetermined and where we have used the identity $\mathbf{K}^{-1} \mathbf{L}=\mathbf{Z}_{m} / \omega$. We can now turn to the calculation of $\mathbf{i}_{1}$ by substituting $\mathbf{v}_{1}$ into (47). This yields

$$
\begin{array}{r}
\mathbf{i}_{1}=\mathbf{Z}_{m}^{-1}\left(e^{-j \mathbf{K} z} \mathbf{P}-e^{+j \mathbf{K} z} \mathbf{Q}\right) \\
-\frac{j \omega}{2} \mathbf{Z}_{m}^{-1} e^{-j \mathbf{K} z} \int_{0}^{z} e^{+j \mathbf{K} z^{\prime}}\left(\mathbf{Z}_{m} \Delta \mathbf{C} \tilde{\mathbf{v}}+\Delta \mathbf{L} \tilde{\mathbf{i}}\right) d z^{\prime} \\
-\frac{j \omega}{2} \mathbf{Z}_{m}^{-1} e^{+j \mathbf{K} z} \int_{0}^{z} e^{-j \mathbf{K} z^{\prime}}\left(\mathbf{Z}_{m} \Delta \mathbf{C} \tilde{\mathbf{v}}-\Delta \mathbf{L} \tilde{\mathbf{i}}\right) d z^{\prime} .
\end{array}
$$

To determine the values of $\mathbf{P}$ and $\mathbf{Q}$ we again have to impose the boundary conditions at $z=0$ and $z=l$

$$
\begin{gathered}
\Delta \mathcal{V}_{1}(z=0)+\mathcal{Z}_{s} \Delta \mathcal{I}_{1}(z=0)=0 \\
\Delta \mathcal{V}_{1}(z=l)-\mathcal{Z}_{L} \Delta \mathcal{I}_{1}(z=l)=0
\end{gathered}
$$

At $z=0$, the result is similar to (30), but with $\mathbf{A}$ and $\mathbf{B}$ replaced by $\mathbf{P}$ and $\mathbf{Q}$ and without source term:

$$
(\mathbf{P}+\mathbf{Q})+\mathbf{Z}_{s} \mathbf{Z}_{m}^{-1}(\mathbf{P}-\mathbf{Q})=0 .
$$

To apply (52), we first need $\mathbf{v}_{1}$ and $\mathbf{i}_{1}$ at $z=l$ :

$$
\begin{array}{r}
\mathbf{v}_{1}(z=l)=e^{-j \mathbf{K} l} \mathbf{P}+e^{+j \mathbf{K} l} \mathbf{Q} \\
-\frac{j \omega}{2} e^{-j \mathbf{K} l}\left[\left(\mathbf{Z}_{m} \mathbf{F}_{+,-}+\mathbf{G}_{+,-} \mathbf{Z}_{m}^{-1}\right) \mathbf{A}\right. \\
\left.+\left(\mathbf{Z}_{m} \mathbf{F}_{+,+}-\mathbf{G}_{+,+} \mathbf{Z}_{m}^{-1}\right) \mathbf{B}\right] \\
+\frac{j \omega}{2} e^{+j \mathbf{K} l}\left[\left(\mathbf{Z}_{m} \mathbf{F}_{-,-}-\mathbf{G}_{-,-} \mathbf{Z}_{m}^{-1}\right) \mathbf{A}\right. \\
\left.+\left(\mathbf{Z}_{m} \mathbf{F}_{-,+}+\mathbf{G}_{-,+} \mathbf{Z}_{m}^{-1}\right) \mathbf{B}\right]
\end{array}
$$

and

$$
\begin{array}{r}
\mathbf{i}_{1}(z=l)=\mathbf{Z}_{m}^{-1}\left(e^{-j \mathbf{K} l} \mathbf{P}-e^{+j \mathbf{K} l} \mathbf{Q}\right) \\
-\frac{j \omega}{2} \mathbf{Z}_{m}^{-1} e^{-j \mathbf{K} l}\left[\left(\mathbf{Z}_{m} \mathbf{F}_{+,-}+\mathbf{G}_{+,-} \mathbf{Z}_{m}^{-1}\right) \mathbf{A}\right. \\
\left.+\left(\mathbf{Z}_{m} \mathbf{F}_{+,+}-\mathbf{G}_{+,+} \mathbf{Z}_{m}^{-1}\right) \mathbf{B}\right] \\
-\frac{j \omega}{2} \mathbf{Z}_{m}^{-1} e^{+j \mathbf{K} l}\left[\left(\mathbf{Z}_{m} \mathbf{F}_{-,-}-\mathbf{G}_{-,-} \mathbf{Z}_{m}^{-1}\right) \mathbf{A}\right. \\
\left.+\left(\mathbf{Z}_{m} \mathbf{F}{ }_{-,+}+\mathbf{G}_{-,+} \mathbf{Z}_{m}^{-1}\right) \mathbf{B}\right] .
\end{array}
$$

Symbols $\mathbf{F}_{+,-}$and $\mathbf{G}_{+,-}$are defined as

$$
\begin{aligned}
& \mathbf{F}_{+,-}=\int_{0}^{l} e^{+j \mathbf{K} z^{\prime}} \Delta \mathbf{C} e^{-j \mathbf{K} z^{\prime}} d z^{\prime}, \\
& \mathbf{G}_{+,-}=\int_{0}^{l} e^{+j \mathbf{K} z^{\prime}} \Delta \mathbf{L} e^{-j \mathbf{K} z^{\prime}} d z^{\prime},
\end{aligned}
$$

where the subindex notation,+- points to the fact that the first exponential under the integral sign has a plus-sign while the second one has a minus-sign. All the other $\mathbf{F}$ and 
G symbols are defined in an analogous way. In an easy to understand notation we rewrite (54) and (55) as

$$
\begin{array}{r}
\mathbf{v}_{1}(z=l)=e^{-j \mathbf{K} l} \mathbf{P}+e^{+j \mathbf{K} l} \mathbf{Q} \\
-e^{-j \mathbf{K} l}\left(\mathbf{T}_{1} \mathbf{A}+\mathbf{U}_{1} \mathbf{B}\right)+e^{+j \mathbf{K} l}\left(\mathbf{T}_{2} \mathbf{A}+\mathbf{U}_{2} \mathbf{B}\right)
\end{array}
$$

and

$$
\begin{array}{r}
\mathbf{i}_{1}(z=l)=\mathbf{Z}_{m}^{-1}\left[e^{-j \mathbf{K} l} \mathbf{P}-e^{+j \mathbf{K} l} \mathbf{Q}\right. \\
\left.-e^{-j \mathbf{K} l}\left(\mathbf{T}_{1} \mathbf{A}+\mathbf{U}_{1} \mathbf{B}\right)-e^{+j \mathbf{K} l}\left(\mathbf{T}_{2} \mathbf{A}+\mathbf{U}_{2} \mathbf{B}\right)\right] .
\end{array}
$$

Applying (52) yields

$$
\mathbf{v}_{1}(z=l)-\mathbf{Z}_{L} \mathbf{Z}_{m}^{-1}\left[\mathbf{Z}_{m} \mathbf{i}_{1}(z=l)\right]=0 .
$$

Finally, (53) and (59) can be combined to determine $\mathbf{P}$ and Q:

$$
\left(\begin{array}{cc}
\phi_{s} & \boldsymbol{\varphi}_{s} \\
\boldsymbol{\varphi}_{L} e^{-j \mathbf{K} l} & \phi_{L} e^{+j \mathbf{K} l}
\end{array}\right)\left(\begin{array}{l}
\mathbf{P} \\
\mathbf{Q}
\end{array}\right)=\left(\begin{array}{cc}
0 & 0 \\
\boldsymbol{\psi}_{A} & \boldsymbol{\psi}_{B}
\end{array}\right)\left(\begin{array}{l}
\mathbf{A} \\
\mathbf{B}
\end{array}\right),
$$

with

$$
\begin{aligned}
\boldsymbol{\psi}_{A} & =e^{-j \mathbf{K} l} \mathbf{T}_{1}-e^{+j \mathbf{K} l} \mathbf{T}_{2}-\mathbf{Z}_{L} \mathbf{Z}_{m}^{-1}\left(e^{-j \mathbf{K} l} \mathbf{T}_{1}+e^{+j \mathbf{K} l} \mathbf{T}_{2}\right) \\
& =\mathbf{V}_{L} e^{-j \mathbf{K} l} \mathbf{T}_{1}-\mathbf{S}_{L} e^{+j \mathbf{K} l} \mathbf{T}_{2}, \\
\boldsymbol{\psi}_{B} & =e^{-j \mathbf{K} l} \mathbf{U}_{1}-e^{+j \mathbf{K} l} \mathbf{U}_{2}-\mathbf{Z}_{L} \mathbf{Z}_{m}^{-1}\left(e^{-j \mathbf{K} l} \mathbf{U}_{1}+e^{+j \mathbf{K} l} \mathbf{U}_{2}\right) \\
& =\mathbf{V}_{L} e^{-j \mathbf{K} l} \mathbf{U}_{1}-\mathbf{S}_{L} e^{+j \mathbf{K} l} \mathbf{U}_{2} .
\end{aligned}
$$

To obtain the second-order perturbation solution, we take the $z$-derivative of (8) taking (9) into account, leading to

$\frac{d^{2} \Delta \mathcal{V}_{2}}{d z^{2}}+\omega^{2}(\tilde{\mathcal{L}} \tilde{\mathcal{C}}) \Delta \mathcal{V}_{2}=-\omega^{2}(\tilde{\mathcal{L}} \Delta \mathcal{C}) \Delta \mathcal{V}_{1}-j \omega \frac{d}{d z}\left(\Delta \mathcal{L} \Delta \mathcal{I}_{1}\right)$

which is similar to (40), but with a more complex source term containing voltages and currents from the first perturbation step instead of unperturbed ones. Following the same procedure as described above for the first perturbation step, the second order perturbation voltages and currents are found. We will not give the explicit expressions for these second order voltages and currents. Similar to the observation made in [19], this second perturbation leads to a substantial gain in accuracy.

\section{VALIDATION EXAMPLES}

\section{A. Frequency domain results}

The theory proposed above for NMTLs is validated by applying it to a ten conductor microstrip line interconnection with random nonuniformities. The nominal structure is shown in Fig. 1. The track width of every line is $w=1.8 \mathrm{~mm}$ and the spacing between any two neighboring lines is $s=700 \mu \mathrm{m}$. The microstrips and ground plane have a thickness $t=35 \mu \mathrm{m}$ and a conductivity $\sigma=5.8 \cdot 10^{7} \mathrm{~S} / \mathrm{m}$. The microstrip lines reside on a Roger's RO4350B substrate with a thickness $h=1.524 \mathrm{~mm}$, a relative permittivity $\varepsilon_{r}=3.66$, and a loss $\operatorname{tangent} \tan \delta=0.003$. The total length of the multiconductor microstrip line is $l=40 \mathrm{~mm}$.

The nominal frequency dependent $\tilde{\mathcal{L}}$ - and $\tilde{\mathcal{C}}$-matrices are obtained with the technique of [20] and [25]. This 2-D electromagnetic numerical technique solves the pertinent complex capacitance and complex inductance problem assuming the quasi-TM behavior of the fields. To model the presence of

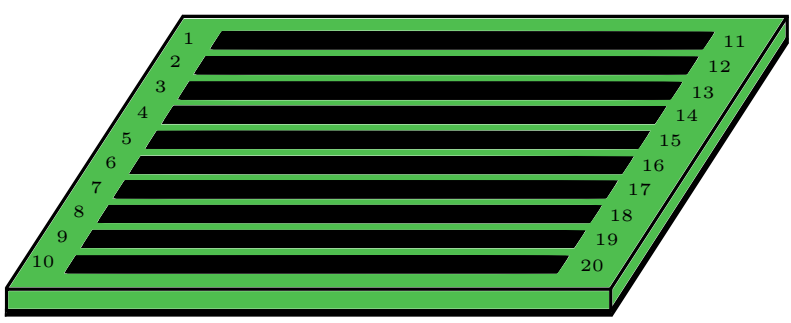

Fig. 1. The nominal uniform microstrip line interconnection with ten signal conductors.

TABLE I

INFLUENCE OF VARYING THE MAXIMAL VALUE OF $\Delta \mathcal{L}$ AND $\Delta \mathcal{C}$

\begin{tabular}{|c||c|}
\hline Max. deviation (\%) & $\Delta S_{12-2} @ 20 \mathrm{GHz}(\%)$ \\
\hline \hline 10 & 0.17 \\
\hline 15 & 0.30 \\
\hline 20 & 0.46 \\
\hline 25 & 0.67 \\
\hline 30 & 0.96 \\
\hline 35 & 1.39 \\
\hline 40 & 2.07 \\
\hline
\end{tabular}

random nonuniformities, the nominal structure is divided in 100 equal sections. Each element of the $\tilde{\mathcal{L}}$ and $\tilde{\mathcal{C}} 10 \times 10$ matrices for any single section is then multiplied with the same random variable (RV) that is uniformly distributed within the interval $[1-\xi, 1+\xi]$. In such a way, we retain perturbed p.u.l. $\mathcal{L}$ and $\mathcal{C}$ matrices that are positive-definite as required for any passive $2 D$ structure. However, for different sections, different RVs are used. The number $\xi$ determines the maximum deviation from the nominal case. We employ the chain parameter matrix approach [2] as a reference solution. In this method the voltages and currents at the input for each individual section are related to the voltages and currents at the output by means of $20 \times 20$ chain parameter matrix. Finally, $S$-parameters can be easily derived from the overall chain parameter matrix obtained as a product of the 100 chain parameter matrices of the individual sections. We compute the $S$-parameters with respect to $50 \Omega$ reference impedances at all ports of the investigated structure. As a sample result, the transmission from port 2 to port 12 is chosen in order to have the transmission through a line which has strong coupling with two neighboring lines. Fig. 2 shows the magnitude of the reflection coefficient $S_{2-2}$, the backward crosstalk $S_{1-2}$, the transmission coefficient $S_{12-2}$ and the forward crosstalk $S_{11-2}$, when the maximum deviation $\xi=25 \%$ with respect to the nominal $\tilde{\mathcal{L}}$ and $\tilde{\mathcal{C}}$ values. As can be seen, the results obtained by applying the perturbation technique are in a very good agreement with the reference method. The phase of the $S$-parameters is also modeled with a very high accuracy. The $S$-parameters for the nominal uniform interconnect are also shown to indicate the influence of random nonuniformities.

To further demonstrate the accuracy and limitations of the perturbation approach, a study of the relative error on the 


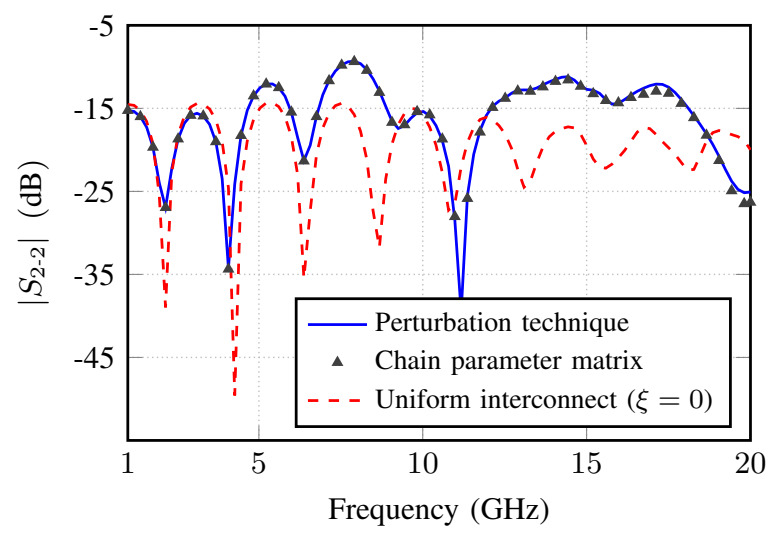

(a)

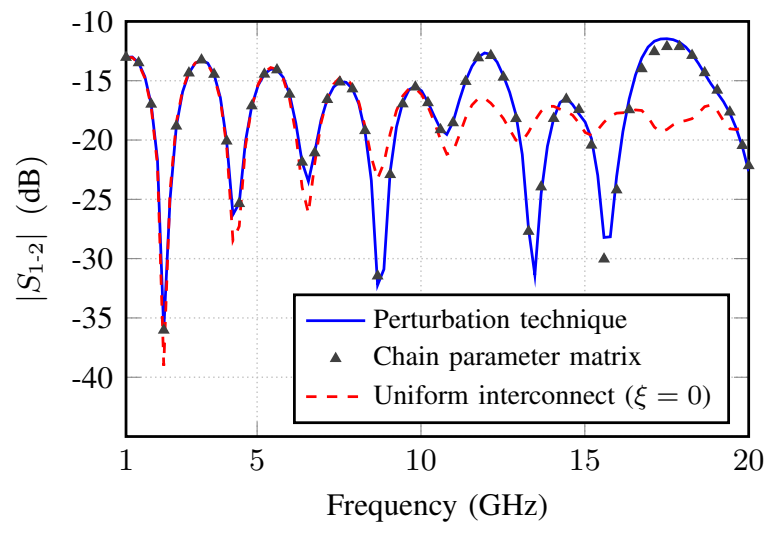

(c)

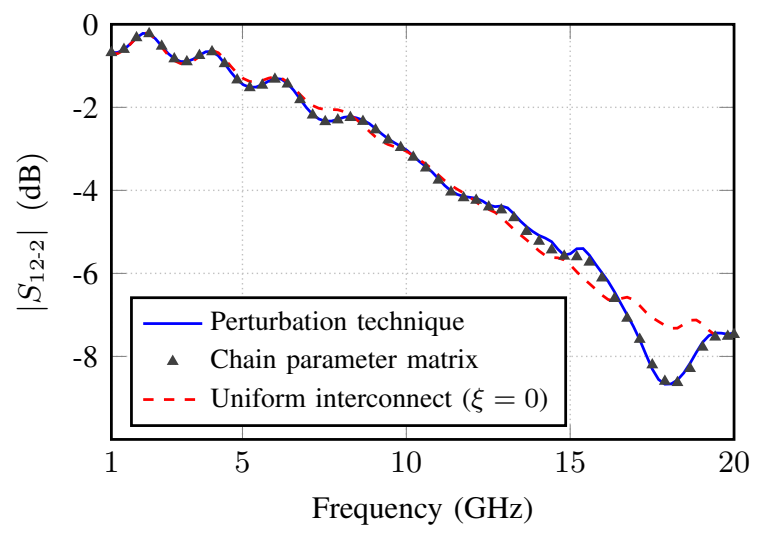

(b)

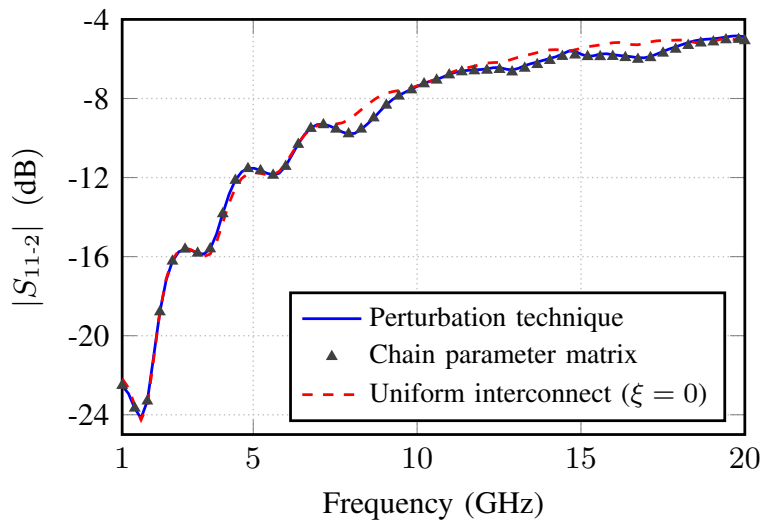

(d)

Fig. 2. $S$-parameters of the ten conductor microstrip line for the case when the maximum variation of the p.u.l. capacitance and inductance is $\xi=25 \%$ using the two-step perturbation and chain parameter matrix techniques. (a) Reflection coefficient $S_{2-2}$. (b) Transmission coefficient $S_{12-2}$. (c) Backward crosstalk $S_{1-2}$. (d) Forward crosstalk $S_{11-2}$. To indicate the influence of the perturbation, the $S$-parameters of the nominal uniform line $(\xi=0)$ are also shown.

transmission coefficient $S_{12-2}$ at the highest frequency of $20 \mathrm{GHz}$ is performed. For different values of the maximum deviation $\xi$, we define the relative error on $S_{12-2}$ taking both magnitude and phase into account as

$$
\Delta S_{12-2}=\left|\frac{S_{12-2}^{(c h)}-S_{12-2}^{(p)}}{S_{12-2}^{(c h)}}\right|,
$$

where $S_{12-2}^{c h}$ and $S_{12-2}^{p}$ are obtained by means of the chain parameter matrix and perturbation techniques, respectively. Table I shows the growing relative error when increasing the maximal values of $\Delta \mathcal{L}$ and $\Delta \mathcal{C}$. However, this error remains limited to $1 \%$ if the perturbations do not exceed $30 \%$ with respect to the nominal case.

Finally, we study the execution time of the code in Matlab 2009a to illustrate the efficiency of the two-step perturbation technique. All calculations were performed on a computer with an Intel Core i7 3630QM Processor and 16 GB of installed memory (RAM). The calculations of the integrals occuring in (49) and (50) determine the computational costs for the perturbation approach. The computational complexity of the reference method is proportional to the number of sections used in concatenation. Table II shows the CPU time for both techniques for 100 frequency samples linearly spaced
TABLE II

CPU TIME COMPARISON

\begin{tabular}{|c|c|c|c|}
\hline $\begin{array}{c}\text { Number of } \\
\text { sections }\end{array}$ & $\begin{array}{c}\text { Perturbation } \\
\text { technique }\end{array}$ & $\begin{array}{c}\text { Reference } \\
\text { solution }\end{array}$ & $\begin{array}{c}\text { Speed-up } \\
\text { factor }\end{array}$ \\
\hline \hline 50 & $7.16 \mathrm{~s}$ & $33.41 \mathrm{~s}$ & 4.67 \\
\hline 100 & $10.83 \mathrm{~s}$ & $62.67 \mathrm{~s}$ & 5.79 \\
\hline 200 & $19.05 \mathrm{~s}$ & $120.08 \mathrm{~s}$ & 6.30 \\
\hline 500 & $41.01 \mathrm{~s}$ & $288.13 \mathrm{~s}$ & 7.03 \\
\hline
\end{tabular}

between 1 and $20 \mathrm{GHz}$ and for a varying number of sections. For example, in the case of 200 sections, the speed-up factor is about 6.3 .

\section{B. Time domain results}

The transient analysis is performed on the high-speed packaging interconnect investigated in [26], which is depicted in Fig. 3. The structure contains six conductors providing an electrical connection between different components on a PCB. The conductors and ground plane are $20 \mu \mathrm{m}$ thick with conductivity $\sigma=5.8 \cdot 10^{7} \mathrm{~S} / \mathrm{m}$. The structure is symmetrical with respect to the dashed straight line depicted in Fig. 3 with a total nominal length $A B$ of $7 \mathrm{~mm}$ measured along 


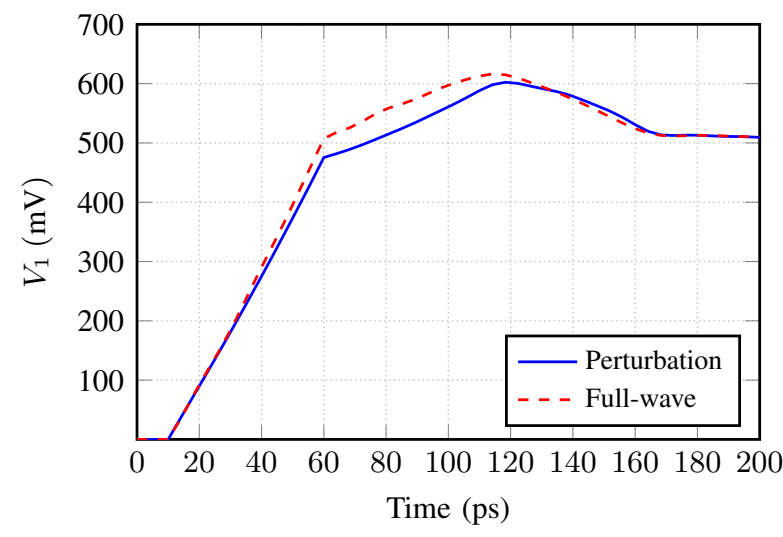

(a)

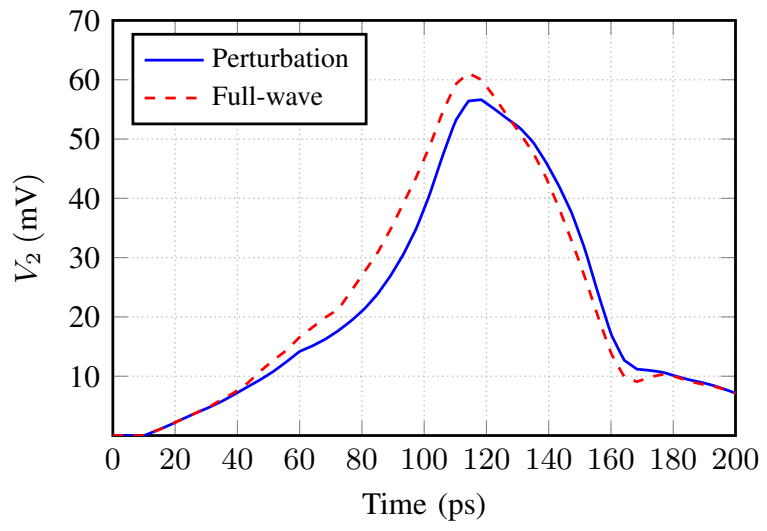

(c)

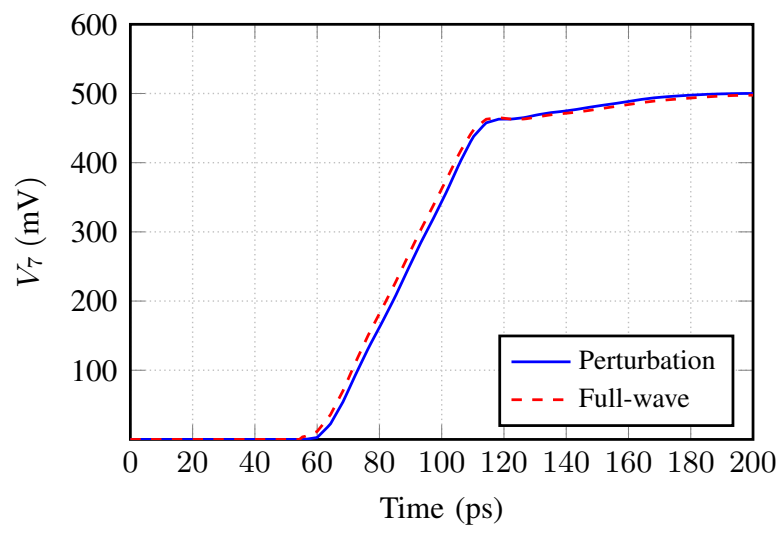

(b)

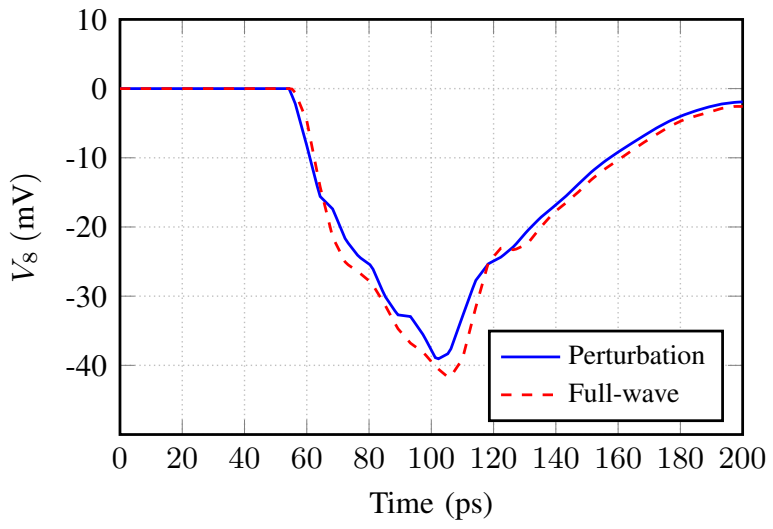

(d)

Fig. 4. Time domain analysis of the high-speed packaging nonuniform interconnect with six signal conductors of Fig. 3. (a) Transient waveform at port 1. (b) Transient response at port 7. (c) Transient response at port 2. (d) Transient response at port 8.

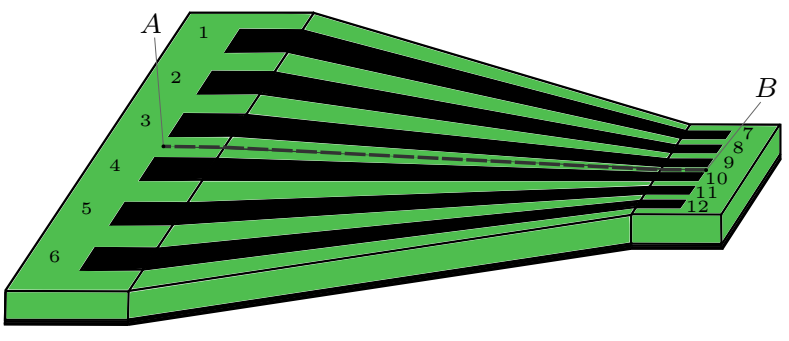

Fig. 3. A high-speed packaging interconnect taper [26].

the dashed central line. The widths and distances between two neighboring conductors are equal to $1 \mathrm{~mm}$ at the left terminations and to $0.125 \mathrm{~mm}$ at the right termination. At both sides, the interconnection structure has $1 \mathrm{~mm}$ long uniform multiconductor line. The interconnect pattern resides on a substrate with a thickness $h=400 \mu \mathrm{m}$, a relative permittivity $\varepsilon_{r}=4.5$, and a loss tangent $\tan \delta=0.001$. Before employing the perturbation technique, the p.u.l. parameters are obtained for nine cross sections orthogonal to the line of symmetry with the method described in [20]. Then, interpolation provides the p.u.l. parameters for the entire structure. Afterwards, we calculate the $S$-parameters applying the two-step perturbation technique to the resulting structure. The numbering of the ports is specified in Fig. 3 . The resulting $12 \times 12 S$-parameters are imported into Agilent's Schematic ADS 2013.06 tool in S12P format for analysis in the time domain. In addition, we perform a full-wave simulation of the investigated structure using Momentum of ADS 2013.06. Then, the results of the full-wave modeling are also used in Schematic to serve as a reference solution. A ramped step signal, going from $0 \mathrm{~V}$ to $1 \mathrm{~V}$ with a rise time of $t_{r}=50 \mathrm{ps}$, is applied to the input port 1 . All ports are matched to $50 \Omega$. Fig. 4 shows the voltages at the input ports 1 and 2 together with the transient response at the output ports 7 and 8 . As can be seen, the results of the perturbation technique represented in time domain are in a very good agreement with the reference fullwave solution. However, the CPU time needed for the transient analysis using the perturbation approach is significantly less than the CPU time needed for the full-wave modeling. In both cases, $S$-parameters were calculated for 100 frequency samples logarithmically spaced in the frequency range from DC to $60 \mathrm{GHz}$. The perturbation technique including calculations of p.u.l. parameters by means of the method described in [20] takes 9 minutes on a computer with Intel(R) Core(TM) Quad CPU Q9650 and 8 GB of installed memory (RAM). In contrast, the full-wave analysis requires about 16 hours 
to perform the same calculations. This clearly defines our perturbation technique as a very efficient one.

\section{CONCLUSion}

In this paper, a two-step perturbation technique has been presented to analyze NMTLs. Nonuniformities were represented as perturbations with respect to a nominal configuration, allowing an interconnect designer to easily see what the effect of (unwanted) perturbations might be. Relying on the Telegrapher's equations, the perturbation approach derives voltages and currents along the multiconductor interconnection from second-order differential equations for the nominal configuration with source terms accounting for the perturbations.

The presented methodology was validated by modeling a ten conductor microstrip line with random uniformities in frequency domain. Compared to a chain parameter matrix approach, excellent accuracy and improved efficiency was achieved. Moreover, a transient analysis performed on a highspeed packaging nonuniform interconnect confirms the validity and very good efficiency of the perturbation method with respect to the full-wave modeling.

\section{APPENDIX A}

\section{EIGENVECTORS OF VOLTAGES AND CURRENTS}

The capacitance and inductance matrices $\tilde{\mathcal{C}}$ and $\tilde{\mathcal{L}}$ are symmetric square $N \times N$ matrices. The eigenvalues $\lambda$ of $\tilde{\mathcal{L}} \tilde{\mathcal{C}}$ are the solutions of $\operatorname{det}(\tilde{\mathcal{L}} \tilde{\mathcal{C}}-\lambda \mathcal{I})=0$ with $\mathcal{I}$ the $N \times N$ unit matrix. The determinant of the transpose of a matrix is identical to the determinant of the matrix itself. As $(\tilde{\mathcal{L}} \tilde{\mathcal{C}})^{T}=\tilde{\mathcal{C}}^{T} \tilde{\mathcal{L}}^{T}=\tilde{\mathcal{C}} \tilde{\mathcal{L}}$, we immediately see that $\operatorname{det}\left[(\tilde{\mathcal{L}} \tilde{\mathcal{C}}-\lambda \mathcal{I})^{T}\right]=\operatorname{det}(\tilde{\mathcal{C}} \tilde{\mathcal{L}}-\lambda \mathcal{I})=0$ and hence we remark that $\tilde{\mathcal{L}} \tilde{\mathcal{C}}$ and $\tilde{\mathcal{C}} \tilde{\mathcal{L}}$ have the same eigenvalues.

Now suppose that $V_{i}$ is an eigenvector of $\tilde{\mathcal{L}} \tilde{\mathcal{C}}$ with eigenvalue $\lambda_{i}$ and $I_{j}$ is an eigenvector of $\tilde{\mathcal{C}} \tilde{\mathcal{L}}$ with eigenvalue $\lambda_{j}$. Consequently,

$$
\begin{aligned}
\left(V_{i}\right)^{T} \lambda_{j} I_{j} & =\left(V_{i}\right)^{T} \tilde{\mathcal{C}} \tilde{\mathcal{L}} I_{j}=\left(\tilde{\mathcal{L}}^{T} \tilde{\mathcal{C}}^{T} V_{i}\right)^{T} I_{j} \\
& =\left(\tilde{\mathcal{L}} \tilde{\mathcal{C}} V_{i}\right)^{T} I_{j}=\left(V_{i}\right)^{T} \lambda_{i} I_{j}
\end{aligned}
$$

For distinct eigenvalues this implies that $\left(V_{i}\right)^{T} I_{j}=0$. Next, we will show that the following orthogonality property holds for two distinct eigenvectors $V_{i}$ and $V_{j}$ :

$$
\left(V_{j}\right)^{T} \tilde{\mathcal{C}} V_{i}=0
$$

The proof runs along the same lines as above. We know that

$$
\tilde{\mathcal{L}} \tilde{\mathcal{C}} V_{i}=\lambda_{i} V_{i}
$$

Hence,

$$
\begin{aligned}
\lambda_{i}\left(V_{j}\right)^{T} \tilde{\mathcal{C}} V_{i} & =\left(V_{j}\right)^{T} \tilde{\mathcal{C}} \tilde{\mathcal{L}} \tilde{\mathcal{C}} V_{i} \\
& =\left(\tilde{\mathcal{L}}^{T} \tilde{\mathcal{C}}^{T} V_{j}\right)^{T} \tilde{\mathcal{C}} V_{i} \\
& =\left(\tilde{\mathcal{L}} \tilde{\mathcal{C}} V_{j}\right)^{T} \tilde{\mathcal{C}} V_{i} \\
& =\lambda_{j}\left(V_{j}\right)^{T} \tilde{\mathcal{C}} V_{i},
\end{aligned}
$$

which implies (A-2) for distinct eigenvalues. Similarly, we have that

$$
\left(I_{j}\right)^{T} \tilde{\mathcal{L}} I_{i}=0
$$

We next prove that $\mathbf{L C}=\mathbf{C L}=\Lambda$. It suffices to prove that $\mathbf{L C}=\Lambda$ as taken the transpose immediately yields the remaining identity. From (20) and (22) we have that

$$
\begin{array}{r}
\mathbf{L} \mathbf{C}=\mathbf{S}^{T} \tilde{\mathcal{L}} \mathbf{S} \mathbf{T}^{T} \tilde{\mathcal{C}} \mathbf{T} \\
=\mathbf{S}^{T} \mathbf{T} \mathbf{T}^{-1} \tilde{\mathcal{L}} \mathbf{S} \mathbf{T}^{T} \mathbf{S} \mathbf{S}^{-1} \tilde{\mathcal{C}} \mathbf{T} .
\end{array}
$$

Using the orthogonality properties $\mathbf{T}^{T} \mathbf{S}=\mathbf{S}^{T} \mathbf{T}=\mathcal{I}_{N}$, (A-6) becomes

$$
\begin{array}{r}
\mathbf{L C}=\mathbf{T}^{-1} \tilde{\mathcal{L}} \mathbf{S S}^{-1} \tilde{\mathcal{C}} \mathbf{T} \\
=\mathbf{T}^{-1} \tilde{\mathcal{L}} \tilde{\mathcal{C}} \mathbf{T}=\mathbf{T}^{-1} \mathbf{T} \Lambda=\Lambda
\end{array}
$$

where we have used (15).

\section{REFERENCES}

[1] B. Curran, I. Ndip, S. Guttowski, and H. Reichl, "A methodology for combined modeling of skin, proximity, edge, and surface roughness effects," IEEE Trans. Microw. Theory Tech., vol. 58, no. 9, pp. 24482455, Sep. 2010.

[2] C. R. Paul, Analysis of Multiconductor Transmission Lines. John Wiley \& Sons, 1994.

[3] J. F. Mao and Z. F. Li, "Analysis of the time response of nonuniform multiconductor transmission lines with a method of equivalent cascaded network chain," IEEE Trans. Microw. Theory Tech., vol. 40, no. 5, pp. 948-954, May 1992.

[4] A. R. Djordjevic and T. K. Sarkar, "Analysis of time response of lossy multiconductor transmission line networks," IEEE Trans. Microw. Theory Tech., vol. 35, no. 10, p. 898908, Oct. 1987.

[5] N. Orhanovic, P. Wang, and V. K. Tripathi, "Time-domain simulation of uniform and nonuniform multiconductor lossy lines by the method of characteristics," IEEE Trans. Comput.-Aided Design Integr. Circuits Syst., vol. 12, no. 6, pp. 900-904, Jun. 1993.

[6] D. Kuznetsov, "Efficient circuit simulation of nonuniform transmission lines," IEEE Trans. Microw. Theory Tech., vol. 46, no. 5, pp. 546 - 550, May 1998.

[7] Q. Xu, Z. F. Li, J. Wang, and J. F. Mao, "Transient analysis of lossy interconnects by modified method of characteristics," IEEE Trans. Circuits Syst. I, vol. 47, no. 3, p. 363375, Mar. 2000.

[8] S. Grivet-Talocia, H. M. Huang, A. E. Ruehli, F. Canavero, and I. M. Elfadel, "Transient analysis of lossy transmission lines: an efficient approach based on the method of characteristics," IEEE Trans. Adv. Packag., vol. 27, no. 1, p. 4556, Feb. 2004.

[9] F.-Y. Chang, "Transient simulation of nonuniform coupled lossy transmission lines characterized with frequency-dependent parameters. I. Waveform relaxation analysis," IEEE Trans. Circuits Syst. I, vol. 39, no. 8 , pp. $585-603$, Aug. 1992.

[10] S. Grivet-Talocia and F. Canavero, "Wavelet-based adaptive solution for the nonuniform multiconductor transmission lines," IEEE Microw. Guided Wave Lett., vol. 8, no. 8, pp. 287-289, Aug. 1998.

[11] S. Barmada and M. Raugi, "Transient numerical solutions of nonuniform MTL equations with nonlinear loads by wavelet expansion in time or space domain,” IEEE Trans. Circuits Syst. I, vol. 47, no. 8, pp. 1178 1190, Aug. 2000.

[12] M. Tong, G. Pan, and G. Lei, "Full-wave analysis of coupled lossy transmission lines using multiwavelet-based method of moments," IEEE Trans. Microw. Theory Tech., vol. 53, no. 7, pp. 2362 - 2370, Jul. 2005.

[13] A. Orlandi and P. C. R., "FDTD analysis of lossy, multiconductor transmission lines terminated in arbitrary loads," IEEE Trans. Electromagn. Compat., vol. 38, no. 3, p. 388399, Aug. 1996.

[14] J. Roden, C. R. Paul, W. Smith, and S. Gedney, "Finite-difference, timedomain analysis of lossy transmission lines," IEEE Trans. Electromagn. Compat., vol. 38, no. 1, pp. 15 - 24, Feb. 1996.

[15] K. Afrooz and A. Abdipour, "Efficient method for time-domain analysis of lossy nonuniform multiconductor transmission line driven by a modulated signal using FDTD technique," IEEE Trans. Electromagn. Compat., vol. 54, no. 2, pp. 482 - 494, Apr. 2012.

[16] H.-H. Chen, "Finite-element method coupled with method of lines for the analysis of planar or quasi-planar transmission lines," IEEE Trans. Microw. Theory Tech., vol. 51, no. 3, pp. 848 - 855, Mar. 2003. 
[17] Q. Xu and P. Mazumder, "Accurate modeling of lossy nonuniform transmission lines by using differential quadrature methods," IEEE Trans. Microw. Theory Tech., vol. 50, no. 10, pp. 2233-2246, Oct. 2002.

[18] M. Tang and J. Mao, "A differential quadrature method for the transient analysis of multiconductor transmission lines," in International Conference on Microwave and Millimeter Wave Technology, Nanjing, P. R. China, 2008, pp. 1423 - 1426.

[19] M. Chernobryvko, D. Vande Ginste, and D. De Zutter, "A two-step perturbation technique for nonuniform single and differential lines," IEEE Trans. Microw. Theory Tech., vol. 61, no. 5, pp. 1758 - 1767, May 2013.

[20] T. Demeester and D. De Zutter, "Quasi-TM transmission line parameters of coupled lossy lines based on the Dirichlet to Neumann boundary operator," IEEE Trans. Microw. Theory Tech., vol. 56, no. 7, pp. 16491660, Jul. 2008

[21] F. Broyde and E. Clavelier, "Multiconductor transmission line models for modal transmission schemes," IEEE Trans. Comp. Packag. Manufact. Technol., vol. 3, no. 2, pp. 306-314, Feb. 2013.

[22] —. (2012, February) Tutorial on echo and crosstalk in printed circuit boards and multi-chip modules. Lecture Slides, Second Edition. Excem. ISBN 978-2-909056-06-7. [Online]. Available: http://www.eurexcem.com/seminar32.htm

[23] L. A. Hayden and V. K. Tripathi, "Nonuniformly coupled microstrip transversal filters for analog signal-processing," IEEE Trans. Microw. Theory Tech., vol. 39, no. 1, pp. 47-53, Jan. 1991

[24] M. Khalaj-Amirhosseini, "Analysis of nonuniform transmission lines using the equivalent sources," Progress In Electromagnetics Research, vol. 71, pp. 95-107, 2007.

[25] T. Demeester and D. De Zutter, "Construction of the Dirichlet to Neumann boundary operator for triangles and applications in the analysis of polygonal conductors," IEEE Trans. Microw. Theory Tech., vol. 58, no. 1 , pp. $116-127$, Jan. 2010.

[26] S. Grivet-Talocia and F. Canavero, "A class of space-expansion methods for the transient simulation of the nonuniform multiconductor transmission lines," in International Symposium on Signals, Systems, and Electronics, Pisa, Italy, 1998, pp. 219-222.

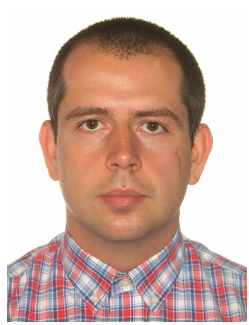

Mykola Chernobryvko was born in 1987 . He received the M.S. degree in electronic engineering from Kharkiv National University of Radioelectronics, Kharkiv, Ukraine, in 2009. He is currently a Doctoral Researcher at the Department of Information Technology at Ghent University. His research interests comprise electromagnetic modeling of interconnects and signal integrity.

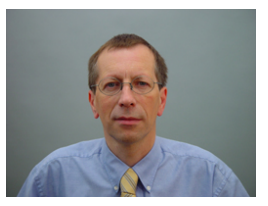

Daniël De Zutter was born in 1953. He received his M. Sc. Degree in electrical engineering from the University of Gent in 1976. In 1981 he obtained a Ph. D. degree and in 1984 he completed a thesis leading to a degree equivalent to the French Aggrgation or the German Habilitation. He is now a full professor of electromagnetics. His research focusses on all aspects of circuit and electromagnetic modelling of high-speed and high-frequency interconnections and packaging, on Electromagnetic Compatibility (EMC) and numerical solutions of Maxwell's equations. As author or co-author he has contributed to more than 200 international journal papers (cited in the Web of Science) and 200 papers in conference proceedings. In 2000 he was elected to the grade of Fellow of the IEEE. He was an Associate Editor for the IEEE Microwave Theory and Techniques Transactions. Between 2004 and 2008 he served as the Dean of the Faculty of Engineering of Ghent University and is now the head of the Department of Information Technology.

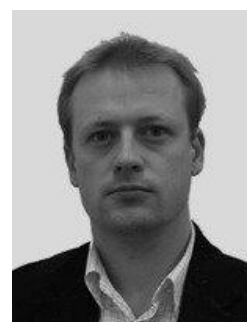

Dries Vande Ginste (SM'12) received the M.S. degree and the Ph.D. degree in electrical engineering from Ghent University, Gent, Belgium, in 2000 and 2005 , respectively. He is currently an Associate Professor with the Department of Information Technology, Ghent University and a Guest Professor at iMinds. In June and July 2004, he was a Visiting Scientist at the Department of Electrical and Computer Engineering, University of Illinois at UrbanaChampaign (UIUC), IL, USA. From September to November 2011, he was a Visiting Professor at the EMC Group, Dipartimento di Elettronica, Politecnico di Torino, Italy. His research interests include computational electromagnetics, electromagnetic compatibility, signal and power integrity, and antenna design. Dr. Vande Ginste was awarded the International Union of Radio Science (URSI) Young Scientist Award at the 2011 URSI General Assembly and Scientific Symposium, the Best Poster Paper Award at the 2012 IEEE Electrical Design of Advanced Packaging and Systems Symposium (EDAPS), the Best Paper Award at the 2013 IEEE Workshop on Signal and Power Integrity (SPI) and the Best Paper Award at the 2013 IEEE International Conference on Electrical Performance of Electronic Packaging and Systems (EPEPS). He served as the co-chair of the 2014 IEEE Workshop on Signal and Power Integrity. He is a Senior Member of the IEEE. 